http://dx.doi.org/10.15407/dopovidi2016.10.042

УдК 539.3

\title{
А.Ю. Глухов
}

Інститут механіки ім. С.П. Тимошенка НАН України, Київ

E-mail: ndrewgl@gmail.com

\section{Вісесиметричні хвилі в шаруватих композитних нестисливих матеріалах з початковими напруженнями при проковзуванні шарів}

(Представлено академіком НАН України О.М. Гузем)

В рамках лінеаризованої теорії пружності для тіл з початковими напруженнями розглянуті постановка та метод розв'язку задач про поширення вісесиметричних хвиль в шаруватих композитних нестисливих заздалегідь напружених матеріалах при проковзуванні шарів. Досліджено випадок поширення хвиль вздовж шарів. Отримано дисперсійне рівняння для квазіпоперечних хвиль та його довгохвильові наближення.

Ключевые слова: шаруватий композитний нестисливий матеріал, початкові напруження, пружні хвилі, дисперсійне рівняння, довгохвильове наближення.

Дослідженням динамічних процесів у тілах з початковими напруженнями, в тому числі і в шаруватих матеріалах, присвячені численні статті в періодичних виданнях [1-5 та ін.]. Їх результати також викладені в ряді монографій [6-8 та ін.] і в оглядових статтях [9, 10].

Такі дослідження є актуальними, так як в реальних пружних тілах, в тому числі й шаруватих композитних матеріалах, майже завжди існують початкові (залишкові) напруження. Природа їх різноманітна. Вони виникають при технологічних процесах створення композитних матеріалів, в елементах конструкцій в результаті технологічних операцій при їх збиранні, в земній корі внаслідок дії геостатичних і геодинамічних сил і т. д. Інколи початкові напруження створюють цілеспрямовано. Вони, взаємодіючи з динамічними напруженнями, істотно впливають на закономірності поширення пружних хвиль.

Поширення плоских хвиль у шаруватих композитних матеріалах періодичної структури 3 початковими напруженнями розглядалося в роботах [1-6]. В [1-3, 6] результати отримані для випадку повного контакту шарів. У статтях $[4,5]$ метод дослідження плоских пружних хвиль у шаруватих композитних матеріалах з початковими напруженнями, викладений в монографії [6], поширений на випадок неповного контакту шарів.

За відсутності початкових (залишкових) напружень аналогічні задачі досліджувались в [11].

У даній роботі в рамках тривимірної динамічної лінеаризованої теорії пружності для тіл 3 початковими напруженнями на основі зазначеного вище методу [6] проведені дослідження поширення вісесиметричних пружних хвиль в шаруватому композитному нестисливому матеріалі з початковими напруженнями при проковзуванні шарів.

(c) А. Ю. Глухов, 2016 
1. Постановка задач і метод досліджень. Розглядається шаруватий композитний нестисливий матеріал з початковими напруженнями, який складається з шарів двох типів, що чергуються.

При дослідженні будемо застосовувати лагранжеві координати $y_{n} \equiv y^{n}$, які в початковому напружено-деформованому стані збігаються з декартовими координатами, і лагранжеві координати $r^{\prime}, \theta, y_{3}$, які в початковому напружено-деформованому стані збігаються з круговими циліндричними координатами.

Декартову систему координат $y_{1}, y_{2}, y_{3}$, в початковому напружено-деформованому стані вибираємо таким чином, щоб вісь $O y_{3}$ була спрямована по нормалі до площин розділу шарів.

Матеріали шарів вважатимемо гіперпружними ізотропними з довільною структурою пружних потенціалів; у разі трансверсально-ізотропних гіперпружних матеріалів шарів будемо вважати, що вісь ізотропії спрямована уздовж вісі $O y_{3}$.

Вважаємо початковий напружений стан однорідним. Також приймаємо, що для кожного 3 шарів мають місце наступні співвідношення

$$
\begin{aligned}
& S_{11}^{0(j)}=S_{22}^{0(j)} \neq S_{33}^{0(j)} ; \quad \sigma_{11}^{0(j)}=\sigma_{22}^{0(j)} \neq \sigma_{33}^{0(j)} ; \\
& \varepsilon_{11}^{0(j)}=\varepsilon_{22}^{0(j)} ; \lambda_{1}^{(j)}=\lambda_{2}^{(j)} ; h^{(j)}=\lambda_{3}^{(j)} h^{(j)} ; j=1,2 .
\end{aligned}
$$

В (1) і нижче індексами в дужках $(j=1,2)$ позначені всі величини, що відносяться до шарів різних типів. Тут $\sigma_{t t}^{0(j)}, S_{t t}^{0(j)}$ то $\varepsilon_{t t}^{0(j)}-$ складові тензора узагальнених напружень, тензора узагальнених напружень Лагранжа і тензора деформацій Гріна відповідно; $h^{(j)}$ i $h^{(j)}-$ товщини $j$-го шару в природному і в початковому напружено-деформованому стані відповідно; $\lambda_{t}^{(j)}-$ коефіцієнти видовження уздовж відповідних вісей.

Як і в [6], приймаємо

$$
\begin{aligned}
& u_{r^{\prime}}^{(j)}=u_{r^{\prime}}^{(j)}\left(r^{\prime}, y_{3}, \tau\right) ; u_{\theta}^{(j)}=0 ; u_{3}^{(j)}=u_{3}^{(j)}\left(r^{\prime}, y_{3}, \tau\right) ; \\
& u_{4}^{(j)} \equiv p^{(j)}=p^{(j)}\left(r^{\prime}, y_{3}, \tau\right) .
\end{aligned}
$$

У цьому випадку в представленні загальних розв'язків просторових динамічних лінеаризованих задач теорії пружності стосовно до загального розв'язку вісесиметричної задачі в циліндричних координатах можна прийняти

$$
\Psi^{\prime(j)} \equiv 0 ; \chi^{\prime(j)}=\chi^{\prime(j)}\left(r^{\prime}, y_{3}, \tau\right)
$$

У розглянутому випадку для визначення переміщень $u^{(j)}$ і складових тензора напружень $Q^{\prime(j)}$ при $y_{3}=$ const отримуємо вирази

$$
\begin{aligned}
& u_{r^{\prime}}^{(j)}=-\frac{\partial^{2}}{\partial r^{\prime} \partial y_{3}} \chi^{(j)} ; u_{3}^{(j)}=\Delta_{1}^{\prime} \chi^{\prime(j)} ; \Delta_{1}^{\prime}=\frac{\partial^{2}}{\partial r^{\prime 2}}+\frac{1}{r^{\prime}} \frac{\partial}{\partial r^{\prime}} ; \rho^{\prime}(j)=\rho(j) ; \\
& u_{4}^{(j)} \equiv p^{(j)}\left[\left(\kappa_{1111}^{\prime(j)}-\kappa_{1133}^{\prime(j)}-k_{1313}^{\prime(j)}\right) \Delta_{1}^{\prime}+\kappa_{3113}^{\prime(j)} \frac{\partial^{2}}{\partial y_{3}^{2}}-\rho^{(j)} \frac{\partial^{2}}{\partial \tau^{2}}\right] \frac{\partial}{\partial y_{3}} \chi^{\prime(j)} ; \\
& Q_{3 r^{\prime}}^{\prime(j)}=\left(\kappa_{1313}^{\prime(j)} \Delta_{1}^{\prime}-\kappa_{3113}^{\prime(j)} \frac{\partial^{2}}{\partial y_{3}^{2}}\right) \frac{\partial}{\partial r^{\prime}} \chi^{\prime(j)} ; \\
& Q_{33}^{\prime(j)}=\left[\left(\kappa_{1111}^{\prime(j)}+\kappa_{3333}^{\prime(j)}-2 \kappa_{1133}^{\prime(j)}-\kappa_{1313}^{\prime(j)}\right) \Delta_{1}^{\prime}+\kappa_{3113}^{\prime(j)} \frac{\partial^{2}}{\partial y_{3}}-\rho^{(j)} \frac{\partial^{2}}{\partial \tau^{2}}\right] \frac{\partial}{\partial y_{3}} \chi^{\prime(j)}
\end{aligned}
$$


Для визначення функцій $\chi^{\prime(j)}$ за умови (3) маємо рівняння

$\left[\left(\Delta_{1}^{\prime}+\xi_{2}^{\prime(j) 2} \frac{\partial^{2}}{\partial y_{3}^{2}}\right)\left(\Delta_{1}^{\prime}+\xi_{3}^{\prime(j) 2} \frac{\partial^{2}}{\partial y_{3}^{2}}\right)-\frac{\rho^{(j)}}{\kappa_{1331}^{\prime(j)}}\left(\Delta_{1}^{\prime}+\frac{\partial^{2}}{\partial y_{3}^{2}}\right) \frac{\partial^{2}}{\partial \tau^{2}}\right] \chi^{\prime(j)}=0$.

Величини $\xi_{2}^{(j) 2}$ і $\xi_{3}^{\prime(j) 2}$ в (6) визначаються так, як і в [6]; $\rho^{(j)}$ - щільність матеріалів кожного $з$ шарів в природному стані.

Таким чином, відповідно до викладеного, дослідження закономірностей поширення вісесиметричних пружних хвиль у шаруватих композитних нестисливих матеріалах з початковими напруженнями зводиться до побудови розв'язків рівняння (6) при задоволенні граничних умов на площинах розділу шарів і умов періодичності Флоке.

Розглянемо поширення вісесиметричної хвилі в радіальному напрямку в шаруватому композитному нестисливому матеріалі з початковими напруженнями. У цьому випадку за аналогією 3 [6] для визначення «істинної» фазової швидкості поширення вісесиметричних хвиль у шаруватому композитному матеріалі з початковими напруженнями приймемо

$$
\chi^{\prime(j)}\left(r^{\prime}, y_{3}, \tau\right)=\chi^{\prime(j)(0)}\left(y_{3}\right) H_{0}^{(1)}\left(r^{\prime} k\right) e^{-i \omega \tau} ; C=\omega k^{-1} ; j=1,2 .
$$

В (7) $k$ і $\omega-$ хвильове число і кругова частота; $C$ - “істинна" фазова швидкість вісесиметричних хвиль; $H_{0}^{(1)}(x)-$ функція Ханкеля нульового порядку першого роду; $\chi^{(j)(0)}-$ амплітудна функція. Надалі індексом (0) позначені всі амплітудні величини в представленнях типу (7).

Підставляючи (7) в (4), для визначення амплітуд переміщень отримуємо такі вирази:

$$
\begin{aligned}
& u_{r^{\prime}}^{(j)(0)}=-\frac{d^{2}}{d y_{3}} \chi^{\prime(j)(0)}\left(y_{3}\right) ; u_{3}^{(j)(0)}=-k^{2} \chi^{\prime(j)(0)}\left(y_{3}\right) ; \\
& p^{(j) 0}=\left[\kappa_{3113}^{\prime(j)} \frac{d^{2}}{d y_{3}^{2}}-k^{2}\left(k_{1111}^{\prime(j)}-\kappa_{1133}^{\prime(j)}-\kappa_{1313}^{\prime(j)}\right)+\omega^{2} \rho^{(j)}\right] \frac{d}{d y_{3}} \chi^{\prime(j)(0)}\left(y_{3}\right) .
\end{aligned}
$$

Аналогічно, підставляючи (7) в (5), для визначення амплітуд складових тензора напружень $Q^{\prime(j)}$ при $y_{3}=$ const маємо

$$
\begin{aligned}
& Q_{3 r^{\prime}}^{\prime(j)(0)}\left(y_{3}\right)=-\left(\kappa_{3113}^{\prime(j)} \frac{d^{2}}{d y_{3}^{2}}+k^{2} \kappa_{1313}^{\prime(j)}\right) \chi^{(j)(0)}\left(y_{3}\right) \\
& Q_{33}^{\prime(j)(0)}\left(y_{3}\right)=\left[\kappa_{3113}^{\prime(j)} \frac{d^{2}}{d y_{3}^{2}}-k^{2}\left(\kappa_{1111}^{\prime(j)}+\kappa_{3333}^{\prime(j)}-2 \kappa_{1133}^{\prime(j)}-\kappa_{1313}^{\prime(j)}\right)+\omega^{2} \rho(j)\right] \frac{d}{d y_{3}} \chi^{\prime(j)(0)}\left(y_{3}\right) .
\end{aligned}
$$

Підставляючи (7) в (6), отримуємо рівняння для визначення функцій $\chi^{(j)(0)}\left(y_{3}\right)$

$$
\left[\left(\xi_{2}^{\prime(j) 2} \frac{d^{2}}{d y_{3}^{2}}-k^{2}\right)\left(\xi_{3}^{(j) 2} \frac{d^{2}}{d y_{3}^{2}}-k^{2}\right)+\frac{\omega^{2} \rho^{(j)}}{\kappa_{1331}^{\prime(j)}}\left(\frac{d^{2}}{d y_{3}^{2}}-k^{2}\right)\right] \chi^{(j)(0)}\left(y_{3}\right)=0 .
$$

Оскільки в (7)-(10) всі співвідношення представлені через амплітудні величини, то умови на границі контакту шарів і умови періодичності також запишемо для амплітудних величин. За умови проковзування при $y_{3}=0$ повинні виконуватися умови:

$$
u_{3}^{\prime(1)(0)}(0)=u_{3}^{\prime(2)(0)}(0) ; Q_{33}^{\prime(1)(0)}(0)=Q_{33}^{\prime(2)(0)}(0) ; Q_{3 r^{\prime}}^{(1)(0)}(0)=0 ; Q_{3 r^{\prime}}^{(2)(0)}(0)=0 .
$$

Відповідно до теореми Флоке також повинні ще виконуватися умови:

$$
\begin{aligned}
& u_{3}^{\prime(1)(0)}\left(h^{(1)}\right)=u_{3}^{\prime(2)(0)}\left(-h^{(2)}\right) ; Q_{33}^{(1)(0)}\left(h^{(1)}\right)=Q_{33}^{\prime(2)(0)}\left(-h^{(2)}\right) ; \\
& Q_{3 r^{\prime}}^{\prime(1)(0)}\left(h^{(1)}\right)=0 ; Q_{3 r^{\prime}}^{\prime(2)(0)}\left(-h^{(2)}\right)=0 .
\end{aligned}
$$


Таким чином, у даному випадку необхідно знайти розв’язок звичайного диференціального рівняння (10), що задовольняє умовам (11) і (12) з урахуванням позначень (8) і (9).

2. Хвилі вздовж нестисливих шарів. Довгохвильове (низькочастотне) наближення. За аналогією з результатами, викладеними в [6], розв’язок рівняння (10) представимо в такій формі:

$$
\begin{aligned}
& \chi^{(j)(0)}\left(y_{3}\right)=\sum_{m=1}^{4} B_{m}^{(j)} e^{i k \alpha_{\theta}^{(j)}\left[y_{3}+(-1)^{j} h^{(j)} / 2\right]} ; \\
& \theta=\delta_{1 m}+\delta_{2 m}+2\left(\delta_{3 m}+\delta_{4 m}\right) ; j=1,2 .
\end{aligned}
$$

В (13) $\alpha_{1}^{(j) 2}$ та $\alpha_{2}^{(j) 2}-$ корені рівняння, яке отримуємо з (10) після перетворень у вигляді

$$
\kappa_{3113}^{\prime(j)} \alpha^{(j)^{4}}-\left[C^{2} \rho^{(j)}-\kappa_{1111}^{\prime(j)}-\kappa_{3333}^{\prime(j)}+2\left(\kappa_{1133}^{\prime(j)}+\kappa_{1313}^{\prime(j)}\right)\right] \alpha^{(j) 2}-\left(C^{2} \rho^{(j)}-\kappa_{1331}^{\prime(j)}\right)=0 .
$$

Для нестисливого шаруватого композитного матеріалу з початковими напруженнями має сенс (за аналогією з результатами [6]) розглядати квазіпоперечну хвилю, яка поширюється вздовж вісі $O r^{\prime}$ і поляризована в площині $r^{\prime} O y_{3}$. Для такої хвилі $u_{r^{\prime}}^{(j)}$ будуть $O r^{\prime}$ антисиметричні, а $u_{3}^{(j)}$ - симетричні щодо середини відповідних шарів.

Для розглянутого випадку в представленні розв’язку у формі (13) для двох сусідніх шарів приймемо наступні залежності:

$$
B_{1}^{(j)}=B_{2}^{(j)} ; B_{3}^{(j)}=B_{4}^{(j)} .
$$

Враховуючи позначення (8) і (9) і підставляючи (13) і (15) в умови (11) і (12), після ряду перетворень отримуємо однорідну систему алгебраїчних рівнянь, з умови існування нетривіаль-

них рішень якої слідує дисперсійне рівняння відносно $C_{S y_{3}}^{2}=\omega k^{-1}-$ швидкості квазіоперечної хвилі вздовж вісі $O r^{\prime}$, поляризованої в площині $r^{\prime} O y_{3}$.

Для довгохвильового (низькочастотного) наближення, обмежуючись одночленною апроксимацією, отримаємо

$$
\begin{aligned}
& C_{S y_{3}}^{2}=\left\{h^{\prime(1)} \kappa_{3113}^{\prime(2)}\left[\kappa_{1313}^{\prime(1)}\left(\kappa_{1111}^{\prime(1)}+\kappa_{3333}^{(1)}-\kappa_{1133}^{\prime(1)}\right)-2 \kappa_{1331}^{\prime(1)} \kappa_{3113}^{\prime(1)}\right]+\right. \\
& \left.+h^{(2)} \kappa_{3113}^{\prime(1)}\left[\kappa_{1313}^{\prime(2)}\left(\kappa_{1111}^{\prime(2)}+\kappa_{3333}^{\prime(2)}-\kappa_{1133}^{\prime(2)}\right)-2 \kappa_{1331}^{\prime(2)} \kappa_{3113}^{\prime(2)}\right]\right\} \times \\
& \times\left[h ^ { \prime ( 1 ) } o ^ { ( 1 ) } \kappa _ { 3 1 1 3 } ^ { \prime ( 2 ) } \left(k_{1313}^{\prime(1)}-\kappa_{3113}^{\prime(1)}+h^{\prime(2)} o^{(2)} \kappa_{3113}^{\prime(1)}\left(\kappa_{1313}^{\prime(2)}-\kappa_{3113}^{\prime(2)}\right]^{-1} .\right.\right.
\end{aligned}
$$

Аналіз рівняння (16) свідчить, що при поширенні хвиль відбувається взаємодія між шарами композиту.

Таким чином, в даній роботі досліджено поширення вісесиметричних хвиль у шаруватих композитних нестисливих матеріалах з початковими напруженнями при проковзуванні шарів. Розглянуто випадок поширення хвиль уздовж шарів. Отримано дисперсійне рівняння для квазіпоперечних хвиль, а також його довгохвильове наближення.

\section{ЦИТОВАНА ЛІТЕРАТУРА}

1. Гузь А.Н., Кхань Л.М. Распространение волн в композитных слоистых материалах с большими начальными деформациями // Прикл. механика. - 1976. - 12, № 1. - С. 3-11.

2. Гузь А.Н., Ситенок Н.А., Жук А.П. Осесимметричные упругие волны в слоистых сжимаемых композитных материалах с начальными напряженими // Прикл. механика. - 1984. - 20, № 7. - С. 20-30.

3. Кхань Л.М. Распространение волн вдоль слоев в слоистых сжимаемых материалах с начальными деформациями // Прикл. механика. - 1977. - 13, № 9. - С. 21-26.

4. Панасюк О.М. Про поширення хвиль в шаруватих композитних стисливих матеріалах з початковими напруженнями при проковзуванні шарів // Доп. НАН України. - 2010. - № 1. - С. 65-70. 
5. Панасюк О.Н. Распространение квазипоперечных волн в слоистых материалах с начальными напряжениями с учетом проскальзывания // Прикл. механика. - 2011. - 47, № 3. - С. 59-66.

6. Гузь А.Н. Упругие волны в телах с начальными (остаточными) напряжениями. - Киев: А.С.К., 2004. -672 c.

7. Гузь А.Н., Бабии С.Ю., Глухов Ю.П. Смешанные задачи для упругого основания с начальными напряжениями. - Saarbrücken: Lambert Acad. Publ., 2015. - 468 c.

8. Гузь А.Н., Жук А.П., Махорт Ф.Г. Волны в слое с начальными напряжениями. - Киев: Наук. думка, 1976. $104 \mathrm{c}$.

9. Гузь А.Н. Упругие волны в телах с начальными (остаточными) напряжениями // Прикл. механика. - 2002. 38, № 1. - С. 35-78.

10. Бабии С.Ю., Гузь А.Н., Жук А.П. Упругие волны в телах с начальными напряжениями // Прикл. механика. 1979. - 15, № 4. - С. 3-23.

11. Бреховских Л.М. Волны в слоистых средах. - Москва: Наука, 1973. - 344 с.

\section{REFERENCES}

1. Guz' A.N., Khanh L.M. Soviet Appl. Mech., 1976, 12, Iss. 1: 1-7.

2. Guz' A.N., Sitenok N.A., Zhuk A.P. Soviet Appl. Mech., 1984, 20, Iss. 7: 589-596.

3. Khanh L.M. Soviet Appl. Mech., 1977, 13, Iss. 9: 868-873.

4. Panasyuk O.M. Dopov. Nac. akad. nauk Ukr., 2010, No 1: 65-70 (in Ukrainian).

5. Panasyuk O.N. Int. Appl. Mech., 2011, 47, Iss. 3: 276-283.

6. Guz'A.N. Elastic waves in bodies with initial (residual) stresses, Kiev: A.S.K, 2004 (in Russian).

7. Guz' A., Babich S., Glukhov Yu. Mixed problems for elastic foundation with initial stresses, Saarbrücken: Lambert Acad. Publ., 2015 (in Russian).

8. Guz' A.N., Zhuk A.P., Makhort F.G. Waves in a Layer with Initial Stresses, Kiev: Nauk. Dumka, 1986 (in Russian).

9. Guz' A.N. Int. Appl. Mech., 2002, 38, Iss. 1: 23-59.

10. Babich S.Yu., Guz'A.N., Zhuk A.P. Soviet Appl. Mech., 1979, 15, No 4: 277-291.

11. Brekhovskikh L.M. Waves in layered media, Moscow: Nauka, 1973 (in Russian).

Надійшло до редакцї 11.02.2016

\section{А.Ю. Глухов}

Институт механики им. С.П. Тимошенко НАН Украины, Киев

E-mail: ndrewgl@gmail.com

\section{ОСЕСИММЕТРИЧНЫЕ ВОЛНЫ В СЛОИСТЫХ КОМПОЗИТНЫХ \\ НЕСЖИМАЕМЫХ МАТЕРИАЛАХ С НАЧАЛЬНИМИ НАПРЯЖЕНИЯМИ ПРИ ПРОСКАЛЬЗЫВАНИИ СЛОЕВ}

В рамках линеаризированной теории упругости для тел с начальними напряженими рассмотрены постановка и метод решения задач о распространении осесимметричных волн в слоистых композитных несжимаемых предварительно напряженных материалах при проскальзывании слоев. Исследован случай распространения волн вдоль слоев. Получено дисперсионное уравнение для квазипоперечных волн и его длинноволновое приближение.

Ключевые слова: слоистый композитный несжимаемый материал, начальные напряжения, упругие волны, дисперсионное уравнение, длинноволновое приближение.

\section{A.Yu. Glukhov}

S.P. Timoshenko Institute of Mechanics of the NAS of Ukraine, Kyiv

E-mail: ndrewgl@gmail.com

\section{AXISYMMETRIC WAVES IN LAMINATED COMPOSITE INCOMPRESSIBLE MATERIALS WITH INITIAL STRESSES UNDER THE SLIPPING OF LAYERS}

The propagation of axisymmetric elastic waves in laminated composite incompressible materials with the slipping of layers is investigated within the framework of a linearized elasticity theory for bodies with initial stresses. The propagation of waves along the layers is considered. The dispersion equation for quasitransversal waves and its long-wave approximation are obtained.

Keywords: laminated composite incompressible material, initial stresses, elastic waves, dispersion equation, long-wave approximation. 